\title{
Pacific
}

Journal of

Mathematics

\section{RESOLUTIONS MODELED ON TERNARY TREES}

\author{
CHRISTOPHER W. STARK
}

Volume $173 \quad$ No. 2 


\title{
RESOLUTIONS MODELED ON TERNARY TREES
}

\author{
Christopher W. Stark
}

Let $\Gamma$ be a discrete group. An extension of Swan's classic technique of grafting chain complexes shows that $\Gamma$ is of type $\mathrm{FP}(\infty)$ if and only if there is an augmented chain complex $C_{*}$ of finitely generated projective $\mathrm{Z} \Gamma$-modules such that each $H_{i}(C)$ is finitely generated over $\mathbf{Z}$.

This paper establishes the $\mathrm{FP}(\infty)$ property for many discrete groups by grafting together chain complexes, extending the construction of [19] from the homologically trivial case to general complexes; all of these splicing or grafting constructions descend from ideas of Swan [21]. We follow [19] in using a tree as the blueprint for our construction; in that paper the underlying tree was an infinite binary tree, while an infinite ternary tree serves as our model in the present case. The resulting finiteness criterion is easily applied, especially in topological settings:

Theorem 3.1. A countable group $\Gamma$ is of type $\mathrm{FP}(\infty)$ if and only if there is an augmented chain complex $C_{*}$ of finitely generated projective $\mathrm{Z} \Gamma$ modules such that the homology groups of $C_{*}$ are finitely generated over $\mathbf{Z}$.

Theorem 4.1. Let $X$ be a connected $C W$ complex of finite type. If $\Gamma$ is the group of covering transformations of a regular covering projection $p: W \rightarrow$ $X$, where $W$ is homotopy equivalent to a complex of finite type, then $\Gamma$ is of type $\mathrm{FP}(\infty)$.

We recall some definitions and set some conventions. All modules are left modules, unless specified otherwise. Let $R$ be a commutative ring with 1. A group $\Gamma$ is of type $\operatorname{FP}(n, R)$ (or "of type $\operatorname{FP}(n)$ over $R$ ") if and only if there is a resolution by projective $R \Gamma$ modules

$$
\cdots \rightarrow P_{i} \rightarrow P_{i-1} \cdots \rightarrow P_{0} \stackrel{\epsilon}{\rightarrow} R \rightarrow 0
$$

of the trivial $\Gamma$-module $R$ such that $P_{i}$ is finitely generated for $i \leq n$. $\Gamma$ is of type $\operatorname{FP}(\infty, R)$ if and only if it is of type $\operatorname{FP}(n, R)$ for all $n$. If $\Gamma$ is of type $\operatorname{FP}(n, \mathbf{Z})$ then $\Gamma$ is said to be of type $\operatorname{FP}(n)$, and this is the version of the notion which is usually studied. 
The first section of this paper discusses grafting or splicing chain complexes to kill homology groups, the second section sets terminology and notation concerning trees, the third proves the main theorem, and the fourth section gives some applications. In [20] the results of this paper are applied to establish characterizations of Poincaré duality groups and virtual Poincaré duality groups.

Spectral sequence arguments can establish similar finiteness theorems but do not seem to give as much control on fine properties, such as growth rates of resolutions. I am grateful to Peter Kropholler and Ross Geoghegan for comments on alternate proofs of some of these results.

For basic information on groups and modules of type $\mathrm{FP}(n)$ and $\mathrm{FP}(\infty)$, see [3] and [5]. A characterization of these classes of groups based on homological limits is discussed in those volumes and in [4], while [6] gives a topological recognition criterion which is quite different from our Theorem 4.1. Another genre of results shows that a group with good rewriting properties must be of type $\mathrm{FP}(\infty)$, including [1] and [7]; [9] proves a result in this vein concerning automatic groups.

Most of this work was done while the author was a guest of the mathematics department at the University of Maryland and revisions were made at the Winterthur Museum and Library; I thank both institutions for their hospitality. I am deeply grateful to Frank Connolly for comments on [19] which led directly to the present paper.

\section{Homological lemmas.}

We review some homological algebra. Recall that all modules are left modules unless specified otherwise. Let $S$ be a ring with 1 and let $C_{*}, D_{*}$ be chain complexes of $S$-modules. The $n$-fold suspension of $C_{*}$, denoted $\Sigma^{n} C_{*}$, is defined by this commutative square at each degree $p$ :

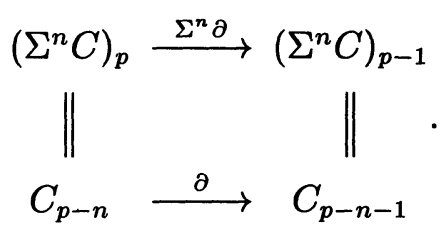

A sign change is sometimes included in the definition of the differentials for the suspension, as in [5], but it will be more convenient here to work with the sign convention above.

If $f: C_{*} \rightarrow D_{*}$ is a chain map then the mapping cone of $f$ is the complex 
$M_{*}=M(f)_{*}$ with

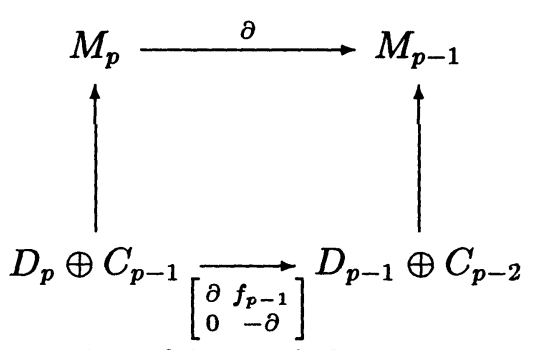

Note that $D_{*}$ is a subcomplex of $M_{*}$ and that a short exact sequence of chain complexes results: $0 \rightarrow D_{*} \rightarrow M(f)_{*} \rightarrow-\Sigma C_{*} \rightarrow 0$, where $-\Sigma C_{*}$ denotes the suspension with all differentials multiplied by $(-1)$.

If $d$ is a non-negative integer then the $d$-skeleton of the chain complex $C_{*}$ is denoted $C_{*}^{(d)}$ and consists of the modules $C_{i}$ for $0 \leq i \leq d$, with the differentials of $C_{*}$.

We recall the following line of argument from [19]:

Let $R$ be a commutative ring with 1 and let $S$ be an augmented $R$ algebra. Let $D_{*}$ be a chain complex of $S$-modules such that $H_{n}(D)$ is a finitely generated $R$-module and $H_{i}(D) \cong 0$ for $n<i<n+d$. Let $C_{*}$ be an $R$-augmented chain complex of projective $S$-modules. There is a chain complex $E_{*}$ such that $E_{i} \cong D_{i}$ for $0 \leq i \leq n, H_{i}(E) \cong H_{i}(D)$ for $0 \leq i<n$, $H_{n}(E) \cong 0$, and $H_{i}(E) \cong H_{i-n-1}(C)$ for $n+1<i<n+d$.

The conclusions of this argument are less important than the main construction, which will be referred to as grafting chain complexes or "splicing chain complexes." The fundamental theorem of homological algebra is the main tool used here, in the following manner.

Pick an epimorphism $\Phi: R^{s} \rightarrow H_{n}(D)$ and let $\left(C^{(d)}\right)^{s}$ denote the direct sum of $s$ copies of the $d$-skeleton of $C$. Consider this commutative diagram:

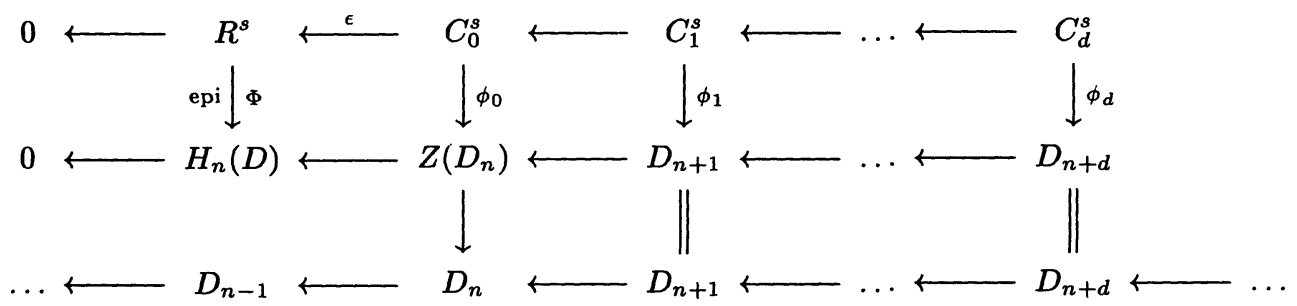

Because $\Sigma^{n}\left(\left(C^{(d)}\right)^{s}\right)$ is a projective complex and the middle row is acyclic, a chain map $\phi_{*}: \Sigma^{n}\left(\left(C^{(d)}\right)^{s}\right) \rightarrow D_{*}$ is induced by $\Phi$. As usual, $\Phi$ determines $\phi_{*}$ up to chain homotopy.

Let $M$ be the mapping cone of $\phi_{*}$ and consider the short exact sequence of chain complexes $0 \rightarrow D_{*} \rightarrow M_{*} \rightarrow-\Sigma^{n+1}\left(\left(C^{(d)}\right)^{s}\right) \rightarrow 0$. The resulting long exact sequence in homology yields the claimed properties, bearing in mind 
that the connecting homomorphism from $H_{n+1}\left(\Sigma^{n+1}\left(\left(C^{(d)}\right)^{s}\right)\right) \cong H_{0}(C)^{s} \cong$ $R^{s}$ to $H_{n}(D)$ is $\phi$.

The next lemma is well known in homological algebra, cf. Proposition 3.1.5 of [2] or Corollary III.5.7 and Exercise III.0.2 of [5].

Lemma 1.1. Let $\Gamma$ be a group and let $R$ be a commutative ring with 1 . If $P$ is a projective $R \Gamma$-module and $M$ is an $R \Gamma$-module which is $R$-free, then $P \otimes_{R} M$ is $R \Gamma$-projective.

Recall that the action of $\Gamma$ on $P \otimes_{R} M$ is diagonal, and observe that if $P$ is a finitely generated $R \Gamma$-projective module and the module $M$ of the Lemma is finitely generated over $R$, then $P \otimes_{R} M$ is also a finitely generated $R \Gamma$-projective module.

Lemma 1.2. Let $\Gamma$ be a group and let $R$ be a commutative ring with 1 . If $M$ is an $R \Gamma-$ module which is finite as a set, then there is an $R \Gamma-$ module $\widetilde{M}$ which is free and finitely generated as an $R$-module and which admits an $R \Gamma$-module epimorphism $\eta: \widetilde{M} \rightarrow M$.

Proof. Let $\widetilde{M}=R^{M}$, the set of all functions $f: M \rightarrow R$. Since $M$ is a finite set, this is a finitely generated free $R$-module, and we give it the left $\Gamma$-action defined by pullbacks: $\gamma \cdot f=\left(\gamma^{-1}\right)^{*} f$, so that $\gamma \cdot f: m \mapsto f\left(\gamma^{-1} m\right)$.

Define

$$
\begin{aligned}
\eta: R^{M} & \rightarrow M \\
f & \mapsto \sum_{m \in M} f(m) m .
\end{aligned}
$$

Since

$$
\begin{aligned}
\eta\left(r_{1} f_{1}+r_{2} f_{2}\right) & =\sum_{m}\left(r_{1} f_{1}+r_{2} f_{2}\right)(m) m \\
& =\sum_{m} r_{1} f_{1}(m) m+\sum_{m} r_{2} f_{2}(m) m
\end{aligned}
$$

and

$$
\begin{aligned}
\eta(\gamma \cdot f) & =\sum_{m}(\gamma \cdot f)(m) m=\sum_{m} f\left(\gamma^{-1} m\right) m \\
& =\sum_{x \in M} f\left(\gamma^{-1} \cdot \gamma x\right)(\gamma x)=\gamma \cdot\left(\sum_{x \in M} f(x) x\right),
\end{aligned}
$$

$\eta$ is an $R \Gamma$-module homomorphism. For each $a \in M$, define

$$
\chi_{a}(m)= \begin{cases}1, & m=a \\ 0, & m \neq a ;\end{cases}
$$

then $\eta\left(\chi_{a}\right)=a$, showing that $\eta$ is an epimorphism. 


\section{Ternary trees.}

We will work with rooted trees of a special type throughout the argument in the next section. Letters from the beginning of the Greek alphabet will be used to denote nodes in graphs below; capital Roman letters will usually refer to graphs or modules. Trees may be infinite.

A ternary tree $T$ is a rooted tree which may be empty; if $T$ is not empty then for each each node $\alpha \in T$ the nodes descended from $\alpha$ must be partitioned into three ternary trees, $L(\alpha), C(\alpha)$ and $R(\alpha)$, the left, central, and right subtrees of $\alpha$. A node is a leaf of the tree if it has no children. A complete ternary tree $T$ is either an empty ternary tree or a nonempty ternary tree with the property that every node which is not a leaf has exactly three children. We will encounter trees in which all nodes but the root and the leaves have exactly three children; such a tree will be called a complete ternary tree with adjoined root.

The depth of the root of a tree is 0 ; the depth of any other node $\alpha$ is the length of the unique path joining $\alpha$ to the root and is denoted $d(\alpha)$.

Nodes in a ternary tree may be represented faithfully as words in the alphabet $\{c, l, r\}$, where a word indicates the path of central advances and left and right turns descending from the root to a node and where the empty word $\epsilon$ denotes the root node. Abusing notation, we take the word representing $\alpha$ as a synonym for $\alpha$. We make a convention that in our ternary trees with adjoined roots, every nonempty word begins with $l$ (i.e. the node of depth one is labeled $l$ ). We let $\alpha(i)$ denote the letter in the $i$-th position of the word representing $\alpha$; put otherwise, the word representing $\alpha$ is $\alpha(1) \alpha(2) \ldots \alpha(d(\alpha))$.

If $\alpha$ and $\beta$ are two nodes in a tree then we define their meet, denoted $\alpha \wedge \beta$, to be the node represented by the maximal common initial string of $\alpha$ and $\beta$. Observe that $\Lambda$ is commutative and associative.

If $\alpha$ and $\beta$ are nodes of a tree then we write $\alpha \preceq \beta$ if and only if $d(\alpha) \leq d(\beta)$ and either

(1) $\alpha=\alpha \wedge \beta$

(2) $\alpha \in L(\alpha \wedge \beta)$ and $\beta \in C(\alpha \wedge \beta) \cup R(\alpha \wedge \beta)$, or

(3) $\alpha \in C(\alpha \wedge \beta)$ and $\beta \in R(\alpha \wedge \beta)$.

Given $\beta$, the nodes $\alpha$ such that $\alpha \preceq \beta$ form a subtree of $\operatorname{depth} d(\beta)$ which is bounded on the right by $\beta$.

If $\alpha$ is a node in a ternary tree $T$ then the collection of nodes represented by the regular expression $\alpha l^{*}$ is the left leg of $T$ through $\alpha$. Similarly, the right leg of $T$ through $\alpha$ is the set of nodes determined by the regular expression $\alpha r^{*}$ and the central leg of $T$ through $\alpha$ is the set of nodes determined by the regular expression $\alpha c^{*}$. 


\section{Resolutions modeled on trees.}

The argument for the next theorem grafts chain complexes repeatedly, using a ternary tree as a blueprint for the construction. The nodes of the tree index submodules in the resolution, in which the sum over all nodes of depth $d$ forms the module of $d$-chains. Some of the morphisms in the construction appear as edges in the tree, but most do not; all the morphisms are conveniently recorded by the order relation $\preceq$ on the tree, however.

Theorem 3.1. A countable group $\Gamma$ is of type $\mathrm{FP}(\infty)$ if and only there is an augmented chain complex $C_{*}$ of finitely generated projective $\mathrm{Z} \Gamma$ modules such that the homology groups of $C_{*}$ are finitely generated over $\mathbf{Z}$.

Proof. If $\Gamma$ is of type $\mathrm{FP}(\infty)$ then a resolution of the trivial $\mathrm{Z} \Gamma$-module $R$ by finitely generated $\mathrm{Z} \Gamma$-projectives exists and has the prescribed properties.

For the converse implication, let $T$ be a ternary tree with adjoined root which is complete of infinite depth. We give a recursive construction of a resolution $P_{*}$ of $\mathbf{Z}$ by finitely generated projective $\mathbf{Z} \Gamma$ modules which associates to each node $\alpha$ of $T$ such a module $P(\alpha)$ and defines $P_{i}:=\oplus_{\alpha: d(\alpha)=i} P(\alpha)$.

A boundary homomorphism will be defined from $P_{i}$ to $P_{i-1}$ so that two conditions are met.

Condition A. If $d(\alpha)=i$ then the image of $P(\alpha)$ lies in $\oplus P(\beta)$, where this sum runs over all nodes $\beta \preceq \alpha$ with $d(\beta)=d(\alpha)-1$.

This convention on boundary homomorphisms implies that for each node $\alpha$ the submodules

$$
S_{i}^{\alpha}:=\oplus_{\beta \preceq \alpha, d(\beta)=i} P(\beta)
$$

of the $P_{i}$ form a subcomplex $S^{\alpha}$, which we describe as the $\alpha$-th skeleton of $P_{*}$. We usually call the morphism

$$
P(\alpha) \rightarrow \oplus_{\beta \preceq \alpha, d(\beta)=d(\alpha)-1} P(\beta)
$$

the $\alpha$-th partial differential.

Condition B. For each righthand node $\alpha$ (i.e. each $\alpha$ such that $\alpha(d(\alpha))=$ $r)$,

$$
H_{d(\alpha)-1}\left(S^{\alpha}\right)=0
$$

Note that this requirement implies that $H_{i}(P)=0$ for all $i>1$ since a direct limit argument shows that $H_{i}(P)=H_{i}\left(S^{\alpha}\right)$ for any node $\alpha$ lying on the right leg through the root and of depth greater than $i$. 
The construction begins with a copy of the chain complex $C_{*}$, arranged as the left leg through the root, i.e. with $P\left(l^{i}\right):=C_{i}$. This initial step is sketched in Figure 1.

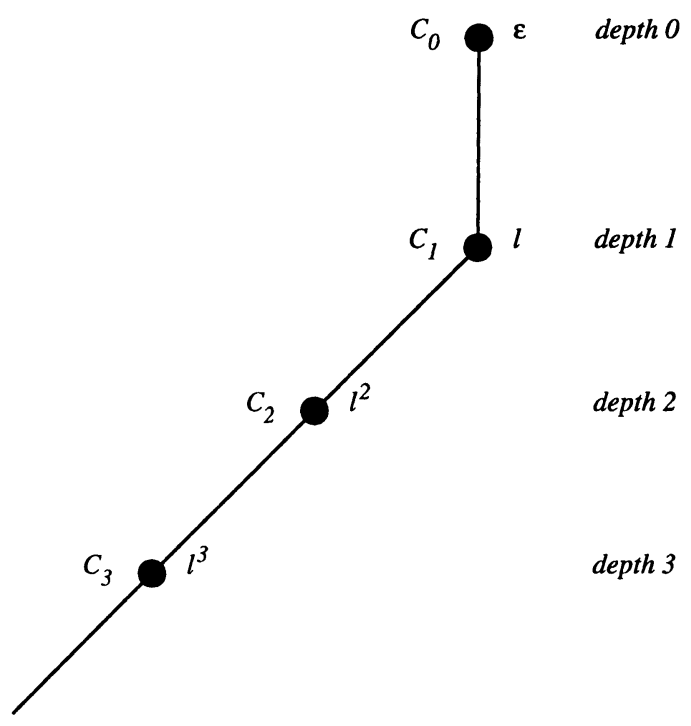

Figure 1. The construction is complete through depth 1 .

At this stage in the construction used in [19], we used a graft to kill $H_{1}(C)$; that argument assumed that the action of $\Gamma$ on $H_{1}(C)$ was trivial and becomes more complicated in the present instance.

Prepare a graft to kill the torsion subgroup $T=\operatorname{Torsion}\left(H_{1}(C)\right)$ by forming the diagram:

0<smiles>[Te][Te]</smiles>

$\mathbf{Z}^{T} \otimes_{\mathbf{Z}} \mathbf{Z}$<smiles>[Te][Te][Te]</smiles>

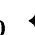
$\mathbf{Z}^{T} \otimes_{\mathbf{Z}} C_{1}$ epi $\downarrow \eta$ $T$

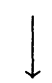

0
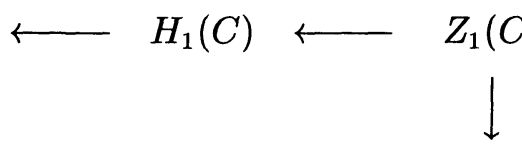

$C_{0}$

$C_{1}$

$C_{2}$<smiles>C=C</smiles>

$C_{2}$

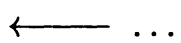

Attach $\mathbf{Z}^{T} \otimes_{\mathbf{Z}} C_{0}$ as the top node $P(l c)$ in the central subtree of $P(l) \cong C_{1}$, with the rest of $\mathbf{Z}^{T} \otimes_{\mathbf{Z}} C_{*}$ descending from $P(l c)$ as the lefthand descending 
path $P\left(l c l^{*}\right)$ in the new subtree. Note that $\eta_{0}: \mathbf{Z}^{T} \otimes_{\mathrm{Z}} C_{0} \rightarrow C_{1}$ is the only new morphism defined so far. Extend our resolution by taking

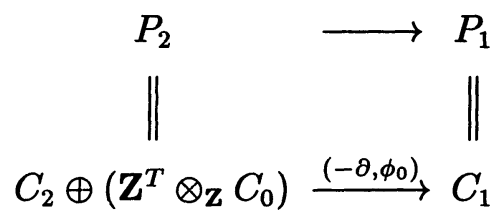

- thus forming an augmented complex $S^{l c}=\left(P_{2} \rightarrow P_{1} \rightarrow P_{0} \rightarrow \mathrm{Z}\right)$ which is finitely generated and projective over $\mathrm{Z} \Gamma$ at each stage, by Lemma 1.1 and which has $H_{1}\left(S^{l c}\right)$ finitely generated and torsion-free over $\mathbf{Z}$. See Figure 2.

Now let $M=H_{1}\left(S^{l c}\right) \cong H_{1}\left(S^{l c}\right) /$ Torsion and prepare a second graft to annihilate this torsion-free quotient of the original homology:

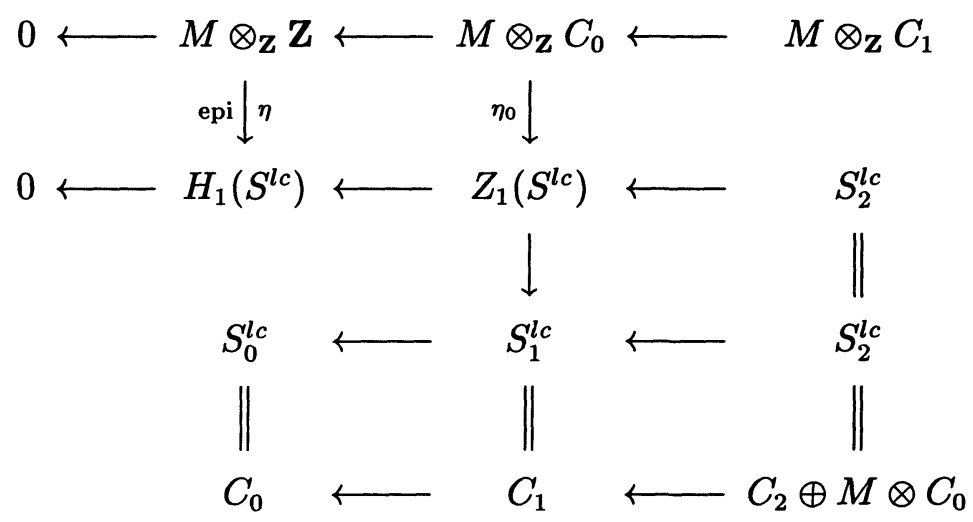

Attach $M \otimes_{\mathbf{z}} C_{0}$ at the righthand node $P(l r)$, with the rest of $M \otimes_{\mathbf{z}} C_{*}$ descending from $P(l r)$ as the lefthand descending path $P\left(l r l^{*}\right)$. We have produced a complex $S^{l r}$ for which $H_{1}\left(S^{l r}\right)=0$.

This completes the construction through depth 2 and we continue by inducing on depth. Our induction hypothesis is that the construction has been completed through depth $d \geq 2$ so that:

(1) for each node $\beta$ of depth $d$ or less, the entire left leg through $\beta$ has been constructed and is a direct sum of copies of $M \otimes_{\mathbf{z}} C_{*}$ (up to suspension, and each choice of the factor $M$ is always a $\mathrm{Z} \Gamma$-module which is a finitely generated free Abelian group),

(2) morphisms satisfying Conditions A and B above have been defined, and

(3) for each righthand node $\alpha$ of depth $d-1$ or less, $H_{d(\alpha)-1}\left(S^{\alpha}\right)=0$.

The nodes of depth $d+1$ are ordered by $\preceq$ and we begin a secondary induction from left to right. The first node of depth $d+1$ is $l^{d+1}$, with $P\left(l^{d+1}\right)=C_{d+1}$ and with morphism $P\left(l^{d+1}\right) \rightarrow P\left(l^{d}\right)$ given by the differential 
of $C_{*}$. Observe that $S^{l^{d+1}}$ is the skeleton $C_{*}^{(d+1)}$.

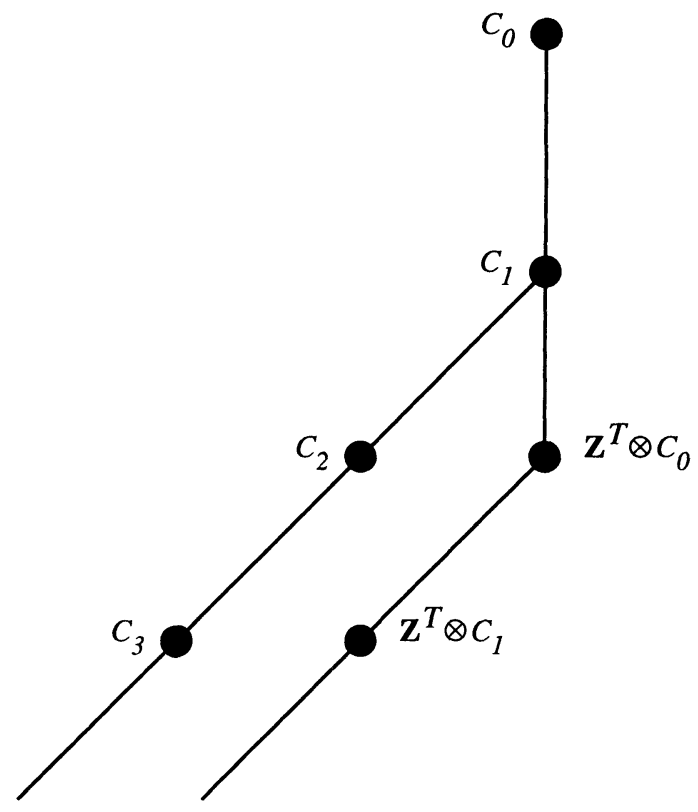

depth 0

depth 1

depth 2

depth 3

Figure 2. The first phase of the construction at depth 2 .

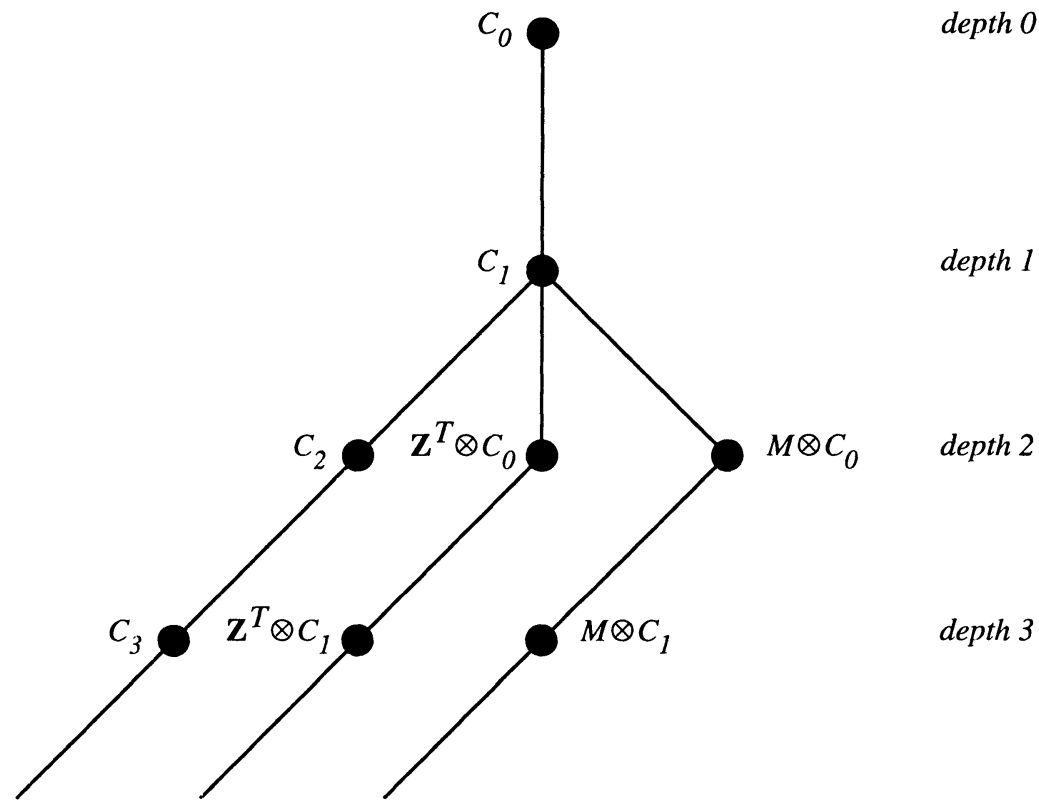

Figure 3. The second phase of the construction at depth 2 .

As we induce from left to right, we fill in the central child $\alpha=\beta c$ of each node $\beta$ of depth $d$ by performing a graft to kill Torsion $\left(H_{d}\left(S^{\gamma}\right)\right)$, where 
$\gamma=\beta l$ is the immediate predecessor at depth $d+1$ of $\alpha$ in the ordering $\preceq$. Righthand children $\alpha=\beta r$ are dealt with by performing a graft to kill the finitely generated, torsion-free quotient $H_{d}\left(S^{\beta c}\right) \cong_{\mathrm{Z \Gamma}} H_{d}\left(S^{\beta} l\right) /$ Torsion. In either case, the graft determines both $P(\alpha)$ and the $\alpha$-th partial differential, as well as the modules $P\left(\alpha l^{j}\right)$. The grafting process establishes properties (1)-(3) for central and righthand nodes, following the discussion of grafting in Section 1.

During this horizontal induction the modules associated to lefthand nodes $\alpha=\beta l$ of depth $d+1$ are determined by continuation from the parent $\beta$. The $\alpha$-th partial differential for a lefthand node other than $l^{d+1}$ is determined (up to chain homotopy) by the fundamental theorem of homological algebra and the homological vanishing requirement for the righthanded node immediately preceding $\alpha$ at depth $d+1$.

\section{Applications.}

Recall that a CW complex $X$ is said to be of finite type if $X$ has a finite number of cells in each dimension. This notion gives a topological setting for the $\operatorname{FP}(\infty)$ property: a finitely presented group $\Gamma$ is of type $\operatorname{FP}(\infty)$ if and only if there is a $K(\Gamma, 1)$ complex of finite type (see [4] or [3], Theorem 1.8). A topological translation of Theorem 3.1 is straightforward:

Theorem 4.1. Let $X$ be a connected $C W$ complex of finite type. If a finitely presented group $\Gamma$ is the group of covering transformations of a regular covering projection $p: W \rightarrow X$, where $W$ is homotopy equivalent to a complex of finite type, then $\Gamma$ is of type $\mathrm{FP}(\infty)$.

Note that the covering space $W$ need not be simply connected, although this result is most easily applied when $W$ is the universal covering space of $X$. The next result shows that some of the hypotheses may be weakened slightly.

Corollary 4.2. Let $X$ be a finitely dominated, connected $C W$ complex and $p: W \rightarrow X$ is a regular covering projection in which $W$ is finitely dominated. If the finitely presented group $\Gamma$ is isomorphic to the group of covering transformations of $p$ then $\Gamma$ is of type $\operatorname{FP}(\infty)$.

Proof. Apply Theorem 4.1 to the covering projection $\widetilde{W} \times S^{3} \rightarrow X \times S^{3}$, in which both spaces are homotopy equivalent to finite complexes by the product formula for Wall's finiteness obstruction [11].

The mixed spaceform problems of topology seek characterizations and classifications of closed manifolds whose universal covers are homotopy equivalent to closed manifolds [18]. Recent progress on the spherical-Euclidean 
spaceform problem of classifying of manifolds whose universal covers are $S^{n} \times \mathbf{R}^{k}$ includes open manifold realization theorems for many groups satisfying appropriate cohomological periodicity conditions [8], [15] and constructions of closed spherical-Euclidean spaceforms whose fundamental groups contain finite dihedral subgroups $[\mathbf{1 3}],[\mathbf{1 7}]$. We also know that the topology of ends of groups constrains the fundamental groups of closed mixed spaceforms [18] and that closed spherical-Euclidean spaceform groups may have infinite virtual cohomological dimension [10].

The results obtained here show that the fundamental groups of closed mixed spaceforms are of type $\mathrm{FP}(\infty)$. In particular, we obtain the following consequence of Theorem 4.1 and the results on virtual Poincaré duality groups from [18].

Corollary 4.3. Let $M$ be a finite Poincaré complex whose universal cover $\widetilde{M}$ has the homotopy type of a Poincaré complex. Let $\Gamma=\pi_{1}(M)$. Then the virtual cohomological dimension $\operatorname{VCD}(\Gamma)$ is finite if and only if $\Gamma$ is a virtual Poincaré duality group.

Proof. A theorem of Gottlieb and Quinn [12], [16] asserts that if $F \rightarrow E \rightarrow B$ is a fibration with finitely dominated fiber, base, and total space, then $E$ is a Poincaré complex if and only if $F$ and $B$ are Poincaré complexes.

We apply this result as in [18], to the fibration classifying $\widetilde{X} \rightarrow X$,

$$
\tilde{X} \rightarrow X \rightarrow B \Gamma,
$$

or rather to the finite-sheeted covering space $X_{0}$ of $X$ corresponding to a finite-index subgroup $\Gamma_{0}$ of $\Gamma$ which has finite cohomological dimension. If $\Gamma_{0}$ is a finitely presented group of type $\mathrm{FP}(\infty)$ and of finite cohomological dimension then Proposition VIII.6.1 and Theorem VIII.7.1 of [5] show that $B \Gamma_{0}$ is finitely dominated. If $\widetilde{X}$ is a Poincaré complex then all three spaces in the fibration $\tilde{X} \rightarrow X_{0} \rightarrow B \Gamma_{0}$ are finitely dominated and the GottliebQuinn theorem shows that $\Gamma_{0}$ shows that $B \Gamma_{0}$ is a Poincaré duality space, i.e. $\Gamma_{0}$ is a Poincaré duality group [14].

Conversely, every virtual Poincaré duality group has finite VCD and is of type VFP, and hence is of type FP( $\infty$ ). (See [5], Sections 8.10 and 8.11, especially remark 2 on p. 222.)

It is a familiar fact that finite-sheeted covering spaces of Poincaré complexes are also Poincaré complexes, but the argument for Corollary 4.3 presented above shows that many infinitely-sheeted covering spaces share this property:

Corollary 4.4. Let $X$ be a finite, connected Poincaré complex and let $p: W \rightarrow X$ be a regular covering projection with a finitely generated group 
$\Gamma$ as the group of covering transformations. If the virtual cohomological dimension $\operatorname{VCD}(\Gamma)<\infty$ then $W$ is finitely dominated if and only if $W$ is a Poincaré complex.

Proof. Poincaré complexes are finitely dominated by definition, so one implication is automatic.

For the converse, take a subgroup $\Gamma_{0}<\Gamma$ of finite index and finite cohomological dimension and consider the diagram

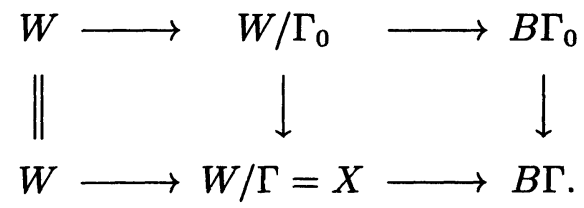

The argument of Corollary 4.3 applies to the top row, in which $W / \Gamma_{0}$ is a Poincaré complex, to show that if $W$ is finitely dominated then $W$ and $B \Gamma_{0}$ are Poincaré complexes.

This result yields a significant restriction in mixed spaceform problems, since we now know that if the fundamental group of a closed, connected manifold $M$ has finite VCD and if the universal cover $\widetilde{M}$ is finitely dominated, then $\widetilde{M}$ is necessarily a Poincaré complex; this is a great constraint on $\widetilde{M}$. One would like to know if the homotopy type of $\widetilde{M}$ is restricted (assuming finite domination) when $\operatorname{VCD}\left(\pi_{1} M\right)$ is infinite, but little evidence seems to be available.

\section{References}

[1] J.M. Alonso, Combings of groups, Algorithms and Classification in Combinatorial Group Theory (G. Baumslag and C.F. Miller III, eds.), MSRI Publications Vol. 23, Springer 1992, 165-178.

[2] D.J. Benson, Representations and Cohomology I, Cambridge University Press, Cambridge 1991.

[3] R. Bieri, Homological Dimension of Discrete Groups, Second Edition, Queen Mary College, University of London, London 1981.

[4] K.S. Brown, Homological criteria for finiteness, Comment. Math. Helv., 50 (1975), 129-135.

[5] C Cohomology of Groups, Graduate Texts in Math., Vol. 87, Springer-Verlag, Berlin-Heidelberg-New York, 1982.

[6] - Finiteness properties of groups, J. Pure and Appl. Alg., 44 (1987), 45-75.

[7] The geometry of rewriting systems: A proof of the Anick-Groves-Squier theorem, Algorithms and Classification in Combinatorial Group Theory (G. Baumslag and C.F. Miller, eds.) III, MSRI Publications, Vol. 23, Springer 1992, 137-163.

[8] F. Connolly and S. Prassidis, Groups which act freely on $\mathbf{R}^{m} \times S^{n-1}$, Topology, 28 (1989), 133-148. 
[9] D.B.A. Epstein, J.W. Cannon, D.F. Holt, S.V.F. Levy, M.S. Paterson and W.P. Thurston, Word Processing in Groups, Jones and Bartlett Publishers, Boston, 1992.

[10] F.T. Farrell and C.W. Stark, Cocompact spherical-Euclidean spaceform groups of infinite VCD, Bull. London Math. Soc., 25 (1993), 189-192.

[11] S.Gersten, A product formula for Wall's obstruction, Amer. J. Math., 88 (1966), $337-346$.

[12] D.H. Gottlieb, Poincaré duality and fibrations, Proc. Amer. Math. Soc., 76 (1979), 148-150.

[13] I. Hambleton and E.K. Pedersen, Bounded surgery and dihedral group actions on spheres, J. Amer. Math. Soc., bf 4 (1991), 105-126.

[14] F.E.A. Johnson and C.T.C. Wall, On groups satisfying Poincaré duality, Ann. of Math., 96 (1972), 592-598.

[15] S. Prassidis, Groups with infinite virtual cohomological dimension which act freely on $\mathbf{R}^{m} \times S^{n-1}$, J. Pure and Appl. Algebra, 78 (1992), 85-100.

[16] F. Quinn, Surgery on Poincaré and normal spaces, Bull. Amer. Math. Soc., 78 (1972), 262-267.

[17] C.W. Stark, Groups acting on $S^{n} \times \mathbf{R}^{k}$ : generalizations of a construction of Hambleton and Pedersen, K-Theory, 5 (1992), 333-354.

[18] - Topological spaceform problems of mixed type, (submitted for publication).

[19] Homologically trivial actions on chain complexes and finiteness properties of infinite groups, J. Pure and Appl. Alg., 94 (1994), 217-228.

[20] - A characterization of virtual Poincaré duality groups, Michigan Math. J., 42 (1995), 99-102.

[21] R.G. Swan, Periodic resolutions of finite groups, Ann. Math., 72 (1960), 267-291.

Received September 19, 1993. Partially supported by NSA Grant MDA904-93-H-3041.

UNIVERSITY OF FLORIDA

GAINESVILLE FL 32611-8000

E-mail address: cws@math.ufl.edu 

Peng Lin and Richard Rochberg, Trace ideal criteria for Toeplitz and Hankel operators on the weighted Bergman spaces with exponential type

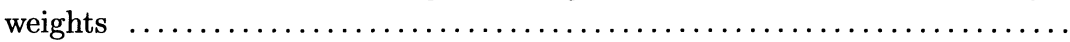

Donald E. Marshall and Arne Stray, Interpolating Blaschke products . .

Kathy D. Merrill and Lynne $\mathbf{H}$. Walling, On quadratic reciprocity over

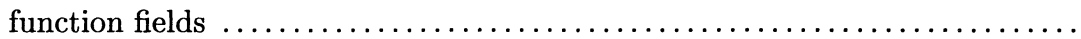

Takahiko Nakazi and Masahiro Yamada, $\left(A_{2}\right)$-conditions and Carleson

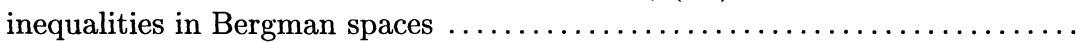

C. Ott, A note on a paper of E. Boasso and A. Larotonda ..............

Victor Patrangenaru, Classifying 3 and 4 dimensional homogeneous Riemannian manifolds by Cartan triples

Carlo Pensavalle and Tim Steger, Tensor products with anisotropic principal series representations of free groups

Ying Shen, On Ricci deformation of a Riemannian metric on manifold with boundary

Albert Jeu-Liang Sheu, The Weyl quantization of Poisson $S U(2) \ldots \ldots$

Alexandra Shlapentokh, Polynomials with a given discriminant over fields of algebraic functions of positive characteristic

Eric Stade and D.I. Wallace, Weyl's law for $S L(3, \mathbb{Z}) \backslash S L(3, \mathbb{R}) / S O(3, \mathbb{R})$

Christopher W. Stark, Resolutions modeled on ternary trees ........ 557

Per Tomter, Minimal hyperspheres in two-point homogeneous spaces .....

Jun Tomiyama, Topological Full groups and structure of normalizers in

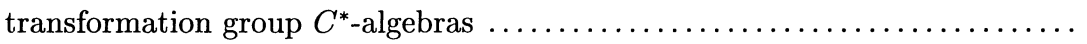

Nik Weaver, Subalgebras of little Lipschitz algebras 


\section{PACIFIC JOURNAL OF MATHEMATICS}

\section{Volume $173 \quad$ No. $2 \quad$ April 1996}

A mean value inequality with applications to Bergman space operators

PATRICK ROBERT AHERn and ZELJKO CUCKOVIC

$H^{p}$-estimates of holomorphic division formulas

MATS ANDERSSON and HASSE CARLSSON

Group structure and maximal division for cubic recursions with a double root

Christian JeAn-Claude Ballot

The Weil representation and Gauss sums

ANTONiA WiLson BLUHER

Duality for the quantum $E(2)$ group

ALFONS VAN DAELE and S. L. WORONOWICZ

Cohomology complex projective space with degree one codimension-two fixed submanifolds 387

KARL HEINZ DOVERMANN and ROBERT D. LITTLE

On the mapping intersection problem

ALEXANDER DRANISHNIKOV

From the $L^{1}$ norms of the complex heat kernels to a Hörmander multiplier theorem for

sub-Laplacians on nilpotent Lie groups

\section{XUAN THINH DUONG}

Isoperimetric inequalities for automorphism groups of free groups

Allen E. Hatcher and Karen Vogtmann

Approximation by normal elements with finite spectra in $C^{*}$-algebras of real rank zero

HUAXIN LIN

Interpolating Blaschke products

DonALD EdDY MARShall and ARNE STRAY

Interpolating Blaschke products generate $H^{\infty}$

JOHN BRADY GARNETT and ARTUR NICOLAU

Classifying 3- and 4-dimensional homogeneous Riemannian manifolds by Cartan triples

VICTOR PATRANGENARU

Polynomials with a given discriminant over fields of algebraic functions of positive

characteristic

ALEXANDRA SHLAPENTOKH

Resolutions modeled on ternary trees

CHRISTOPHER W. STARK

Topological full groups and structure of normalizers in transformation group $C^{*}$-algebras 